\title{
Multimodal imaging of the breast to retrieve the reference state in the absence of gravity using finite element modeling
}

\author{
Remi Salmon 1,*, Thanh Chau Nguyen ${ }^{1,2}$, Linda W. Moore ${ }^{3}$, Barbara L. Bass ${ }^{3}$, Marc \\ Garbey $^{1}$ \\ ${ }^{1}$ Center for Computational Surgery, Houston Methodist Research Institute, Houston, TX, USA \\ ${ }^{2}$ Center for Advanced Computing \& Data Systems, University of Houston, Houston, TX, USA \\ ${ }^{3}$ Department of Surgery, Houston Methodist, Houston, TX, USA \\ rsalmon@houstonmethodist.org
}

\begin{abstract}
We introduce in this work a new method of retrieving the reference state of the breast in a stress-free configuration and estimating at the same time the elasticity of the breast tissues by combining MRI and surface imaging data and using finite element analysis. This reference state of the breast is particularly useful in predicting the cosmetic outcome and the healing process of breast cancer surgery, and breast conserving therapy in particular.
\end{abstract}

Keywords: Breast cancer, breast conserving therapy, multiscale model, finite element analysis, reference state

\section{Introduction}

Breast cancer is the most prevalent form of cancer affecting women, accounting for $26 \%$ of cancer cases in the United States [1]. Additionally, breast cancer survival rate has been increasing during the past decades thanks to advances in early detections, mammography and general breast cancer awareness [2]. This increase in early detections and survival rate of breast cancer has resulted in the development of Breast Conserving Therapy (BCT) that combines localized breast cancer surgery with radiation and adjuvant therapy. By trying to preserve the contour of the breast, BCT improves the quality of life of the patient after surgery [3,4], without impacting the cancer survival rate $[5,6]$. The cosmetic outcome of $\mathrm{BCT}$ remains however less than optimal in many of the cases $[7,8]$.

In order to predict and help improve the cosmetic outcome of BCT we have developed a multiscale model that combines a finite element analysis of the deformation of the soft tissues of the breast after surgery at the macroscopic scale with a biological model of wound healing, operating at the spatial and time scale of a cell cycle. In our wound healing model, we assume that the breast is in a reference state defined as the breast geometry free of mechanical stresses, i.e. in the absence of gravity. We assume in other words that the topology of the cellular matrix can be modelized with a regular hexagonal grid in a fixed frame of reference that is not sensitive to the effect of gravity. We neglect as well the residual internal stresses resulting from the inversion of the

adfa, p. 1, 2011.

(C) Springer-Verlag Berlin Heidelberg 2011 
gravity using finite element analysis. In this gravity-free state, the mechanical stress and strain present in the breast under the effect of gravity is taken into account in the probability of cellular division during the production of scar tissue $[9,10]$.

This reference state of the breast serves as a basis to our model by defining the "unloaded" frame of reference where the biological model of wound healing operates to model the closure of the wound over time. We also use this reference state to compute the contour of the breast and the stress distribution under the effect of gravity in a "loaded" frame of reference. The scheme of Fig. 1 summarizes these two frames of reference and how they interconnect in our multiscale model.

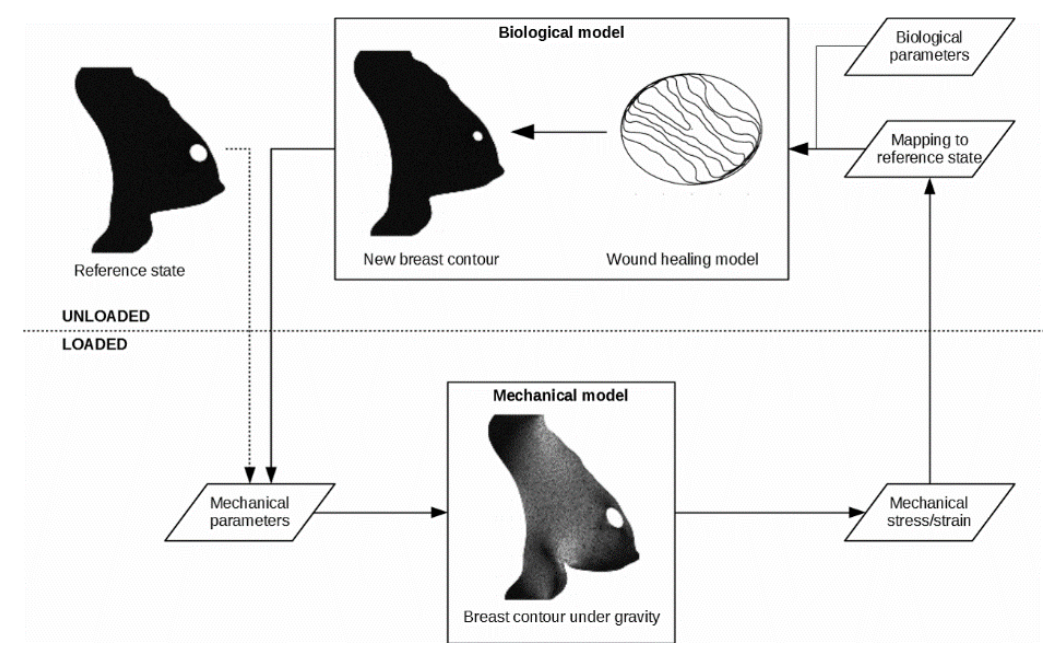

Fig. 1. Flowchart of the multiscale model of BCT, from [10]. The reference state of the breast (top left) is used as a basis to both define the gravity-free "unloaded" frame of reference where the healing model operates and to compute the effect of the gravity in the "loaded" frame of reference.

We have verified the feasibility of our model with a case study of a patient presenting an ideal tumor configuration in the center of the breast, where the reference state of the breast was retrieved from pre-operative MRI imaging only [10]. However the large variability in the anatomy of the patients enrolled in our clinical trial has shown the limitation of MRI imaging as a unique data source in our reconstruction of the reference state of the breast. Indeed, during the MRI acquisition the breast is often compressed onto the MRI coils, resulting in artifacts and missing information in the MRI data $[11,12]$. We have also looked into using pre-operative surface imaging of the breast as input data to retrieve the reference state of the breast. However surface imaging of the breast fails to capture the whole breast contour when the data is acquired with the patients in a standing or sitting position. Indeed the breast is resting on the abdomen and the lower surface of the breast is not visible [13].

To account for this missing ground truth data in our model due to either i) the compression of the breast on the MRI coils and ii) the hidden surface of the breast when the patient is standing up, we present here a method to combine the pre-operative MRI 
imaging with the 3D surface imaging of the breast in order to improve the reconstruction of the reference state of the breast.

\section{Methods}

Determining the reference state of the breast is not a new issue, and previous studies by Rajagopal et al. have looked at retrieving the reference state of a finite element model of the breast using an iterative optimization algorithm [14]. The same technique has been used to perform multimodal image registration between MRI and mammography data [15].

In order to retrieve the correct, patient-specific geometry and elastic modulus of the breast, we combine pre-operative MRI imaging of the breast with a pre-operative 3D surface imaging of the breast, acquired using a Microsoft Kinect device. The reconstruction and generation of the finite element model of the breast from the MRI data is detailed in our previous work $[9,10]$. The data collected with the Kinect device is processed using the RecFusion software in order to reconstruct a 3D surface mesh of the breast [16]. The Kinect surface reconstruction and the MRI data of a patient enrolled in our clinical trial is shown in Fig. 2; this data is then processed to isolate the 3D surface of the breast only.

The method, we propose here, makes use of multimodal imaging to retrieve both the missing boundary conditions in the reference state of the breast reconstructed from the pre-operative data as well as an estimation of the elastic modulus of the breast tissues. We show in the results section an application of our method on a 2D and a 3D model of the breast of the same patient enrolled in a clinical trial currently underway at the Houston Methodist hospital [17].

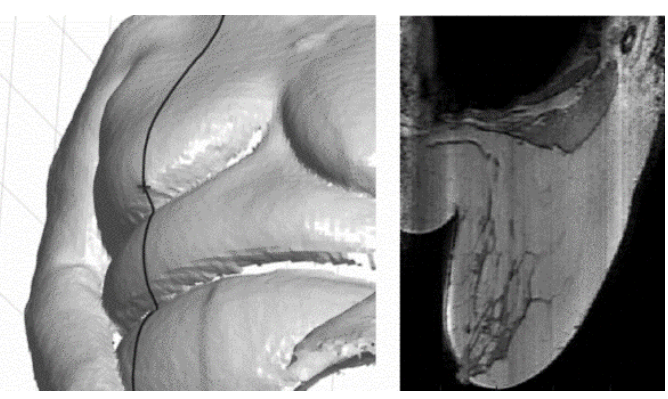

Fig. 2. Kinect Surface reconstruction of the patient acquired before surgery (left). The sagittal plane going through the nipple is drawn over the surface mesh (solid line). MRI image of the same patient in the same sagittal plane, acquired in the prone position (right).

\subsection{Two-dimensional simplification}

We first consider in this study a 2D section of the breast in the sagittal plane going through the nipple for both the MRI and the Kinect surface data, assuming no displacement of the breast in the direction orthogonal to the sagittal plane. We inverse the effect 
of the gravity on the breast model by applying a body force opposite to the one of the gravity, see Fig. 3, using the finite element analysis software FEBio with no displacement boundary conditions on the top, bottom and back surfaces of the breast model [18]. As stated, we neglect here the residual internal stress in the breast after inversion of the gravity. We have developed on this subject an optimization algorithm to compensate for the residual internal stress detailed in Thanoon et al. [19]. We use a uniform hyperelastic Neo-Hookean material to model the breast tissues, parameterized in FEBio by its Young's modulus $E$ and a density $v$. We fix the density in this study to $v=0.49$, assuming the breast tissue be quasi-incompressible.

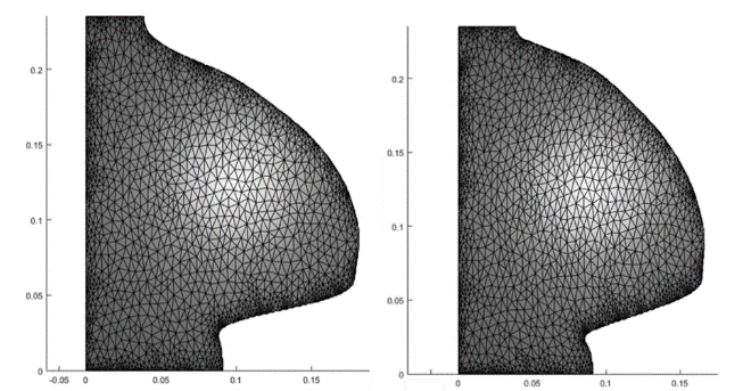

Fig. 3. 2D mesh of the breast reconstructed from the MRI data $A$ (left), and result after inversion of the gravity $A^{*}$ (right). Axis in meters.

Let us name $A=\left\{a_{i}\right\}$ the curve defining the skin envelope of the breast, i.e. the breast contour, retrieved from the MRI data acquired with the patient in the prone position, with $a_{i}=\left\{x_{i}, y_{i}\right\}, i \in[1, N]$. We note $A^{*}=\left\{a_{i}^{*}\right\}$ the breast contour $A$ after inversion of the effect of gravity, where $A^{*}$ is a function of the unknown Young's modulus $E$. Similarly, we name $B=\left\{b_{i}\right\}$ the skin envelope of the breast retrieved from the Kinect surface data, with $b_{i}=\left\{x_{i}, y_{i}\right\}, i \in[1, M]$.

Due to the fact that, for most patients enrolled in our clinical study, the breast of the patient is resting on the abdomen when the patient is standing up during the acquisition of the 3D surface imaging, as seen in Fig. 2, it is actually not possible to retrieve entirely the contour of the breast. To virtually recover this missing contour of the breast, we define the unknown lower extremity of the breast contour in the sagittal plane $b_{B}=$ $\left\{x_{B}, y_{B}\right\}$ such that the curve going through $\left\{b_{i}, b_{B}\right\}$ is the contour of the entire breast. In practice, we define a sampling of the space of the 2D sagittal plane where $b_{B}$ is a priori located, see Fig. 4. For each possible $b_{B}^{k}, k \in[1, K]$, where $K>1$, we define a new curve $C^{k}=\left\{c_{i}\right\}$, interpolation of the parametric curve $\left\{b_{i}, b_{B}^{k}\right\}$ on a regular interval of $M^{*}>M$ points, with $c_{i}=\left\{x_{i}, y_{i}\right\}, i \in\left[1, M^{*}\right]$. The curve $C^{k}$ is computed using the piecewise cubic Hermite interpolating polynomial function of MATLAB in order to ensure smoothness and $\mathrm{C} 1$ continuity. We fix $M^{*}=150$ data points. The upper, lower and back sides used for the no displacement boundary conditions are created artificially in MATLAB in order to close the 2D breast contour. Finally, we name $C^{k *}=\left\{c_{i}^{*}\right\}$ the breast contour $C^{k}$ after inversion of the gravity on the breast model. 

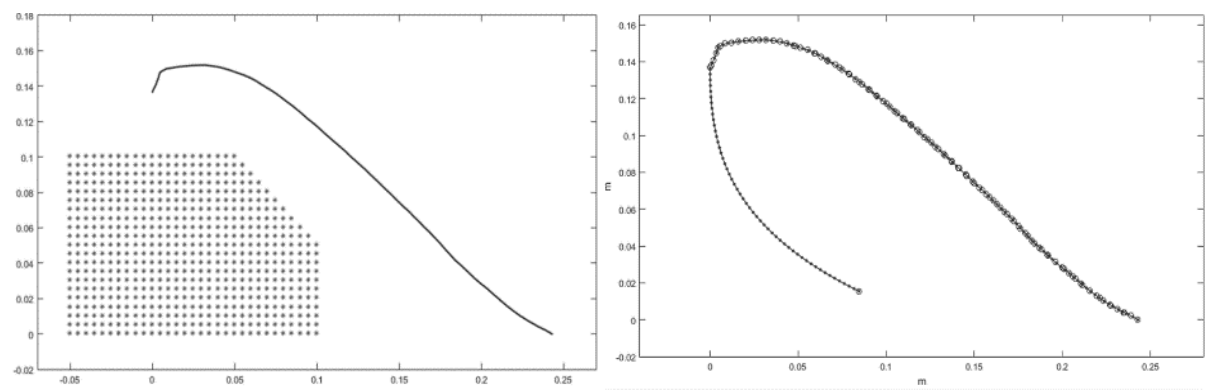

Fig. 4. Skin envelope of the breast $B$ acquired from surface imaging (solid line, where the top of the breast is on the right end and the bottom on the left end) and sampling of the 2D space for the possible location of the $k$ possible lower extremum points $b_{B}^{k}$ (left). This particular grid of $b_{B}^{k}$ points is chosen in order for the finite element simulation of the inversion of the gravity to converge without error from the solver. Sample of a generated 2D breast contour (right). Axis in meters.

In order to retrieve the unknown parameters of the model $E$ and $b_{B}^{k}$, we minimize an objective function $f$ defined as the average distance between the two breast contours in the absence of gravity $A^{*}$ and $C^{k *}$ :

$$
f(E, k)=\frac{1}{M^{*}} \sum_{i}\left\|\tilde{a}_{i}^{*}(E)-c_{i}^{*}(E, k)\right\|
$$

where $\tilde{a}_{i}^{*}$ is the interpolation of $a_{i}^{*}$ on the regular interval defined previously for $c_{i}^{*}$. The minimum $\left(E_{\text {min }}, k_{\text {min }}\right)$ of the objective function is such that $A^{*}\left(E_{\min }\right)$ is the estimated reference state of the breast in our multiscale model. We show in Fig. 5 a diagram to illustrate the workflow leading to the evaluation of the objective function and the optimization of the parameters of our model.

\subsection{Three-dimensional formulation of the problem}

We develop a similar method on a 3D mesh of the breast composed of a number $S$ sagittal slices equally spaced for both the MRI and the Kinect surfaces of the breast. For those two datasets, each curve of the breast contour surface is closed using top, bottom and back surface boundaries identical to the ones shown in Fig. 3, with no displacement boundary conditions. From the resulting 3D cloud of points of the combined slices, we first generate a surface mesh using the Poisson surface reconstruction algorithm implemented in Meshlab [20]. Finally, a 3D volumetric mesh using tetrahedral volume elements is generated using the Gmsh software [21].

Following the same methodology, we name $A_{j}=\left\{a_{i}^{j}\right\}$ the collection of $S$ slices defining the surface of the breast retrieved from the MRI data acquired with the patient in the prone position, with $a_{i}^{j}=\left\{x_{i}, y_{i}, z_{i}\right\}, i \in\left[1, N_{j}\right]$ and $j \in[1, S]$. We note $A_{j}^{*}=\left\{a_{i}^{j *}\right\}$ the slice $A_{j}$ after inversion of the effect of gravity on the 3D model of the breast. Similarly, we name $B_{j}=\left\{b_{i}^{j}\right\}$ collection of $S$ slices defining the surface of the breast retrieved from the Kinect surface data, with $b_{i}{ }^{\prime}=\left\{x_{i}, y_{i}, z_{i}\right\}, i \in\left[1, M_{j}\right]$ and $j \in[1, S]$. 
In order to reduce the number of unknown parameters in the $3 \mathrm{D}$ reconstruction of the breast surface from the Kinect data, we use the follow technique: we define the new unknown parameter point that $b_{B}^{k}, k \in[1, K]$ such that $b_{B}^{k}$ is the projection of $b_{M_{S}}^{S}$ on the belly surface of the patient extracted from the Kinect data and interpolated under the surface of the breast, where $s$ is the sagittal slice going through the nipple, see Fig. 2 (left). The degrees of freedom of $b_{B}^{k}$ are then reduced to one by constraining the point $b_{B}^{k}$ to belong to the interpolated belly surface of the patient. The curve $L$ defined by the spline interpolation between the points $\left\{b_{M_{1}}^{1}, b_{B}^{k}, b_{M_{S}}^{S}\right\}$ is the lower boundary of the breast, initially missing from the Kinect surface imaging. Let us note $l_{k}^{j}$ the intersection of the sagittal plane of the slice $j$ and the curve $L$. We define a new collection of slices $C_{j}^{k}=\left\{c_{i}^{j}\right\}$, interpolation of the parametric curve $\left\{b_{i}^{j}, l_{k}^{j}\right\}$ on a regular interval of $M^{*}$ points. Finally, the same algorithm for the evaluation of the objective function is applied; a diagram Fig. 5 summarizes the data workflow leading to the evaluation of the objective function $f$.

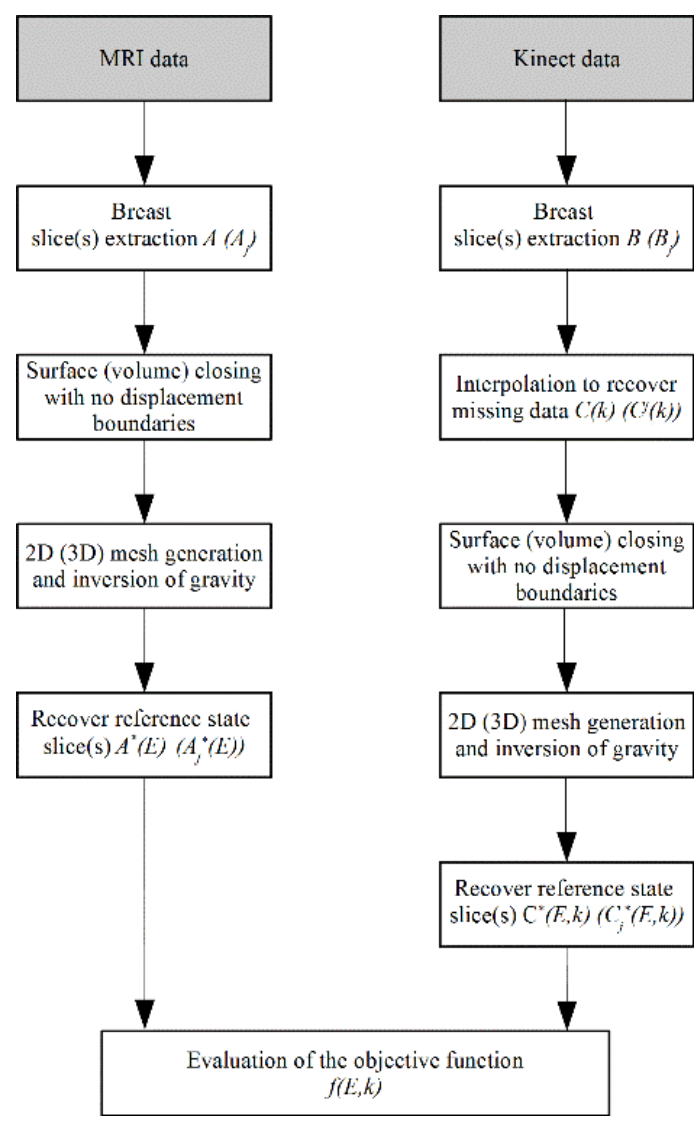

Fig. 5. Data workflow leading to the construction of the objective function $f$. The variable defined for the $3 \mathrm{D}$ formulation of the problem are noted between parentheses. 


\section{Results}

We show here the result of our method on a 67 years old patient enrolled our clinical study, diagnosed with an invasive dual carcinoma on the lower inner quadrant of the right breast. Post-operative MRI and Kinect surface imaging were acquired at the Houston Methodist hospital. The MRI data was acquired with a voxel resolution of $07031 \times 0.7031 \times 1 \mathrm{~mm}$, and the Kinect surface data was acquired with an average resolution of $1.2 \mathrm{~mm}$. For both data sets, a 2D triangular mesh and 3D tetrahedral mesh are generated with an average resolution of $2 \mathrm{~mm}$.

In the 2D formulation of our problem, the objective function $f$ defined in Eq. (1) was evaluated with a Young's modulus $E$ ranging from $0.2 \mathrm{kPa}$ to $7.5 \mathrm{kPa}$ with a resolution of $0.1 \mathrm{kPa}$ from 0.2 to $1 \mathrm{kPa}$ and a resolution of $0.5 \mathrm{kPa}$ from 1 to $7.5 \mathrm{kPa}$ and a total number of 19 values; $b_{B}$ was chosen on a grid of 596 points on a regular grid of $5 \mathrm{~mm}$ resolution, for a total of $19 * 596$ simulation runs. The minimum of the objective function is obtained for $E_{\min }=0.6 \mathrm{kPa}$, within the range of measured values for fat and glandular breast tissues in the literature [22]. We observe indeed on Fig. 2 (right) that the breast appears to have a large proportion of fat tissues that correlates with a lower Young's modulus.

The fitting between the breast contours reconstructed from MRI data and surface imaging of the breast after optimization of the objective function is shown in Fig. 6 (left). We observe a relatively good fit for the upper, "visible" section of the breast contour with an error within in the order of magnitude of the mesh resolution of $2 \mathrm{~mm}$. The less optimal fit of the lower section of the breast can be explained by the use of interpolation where the surface imaging of the breast initially failed to capture the breast contour.

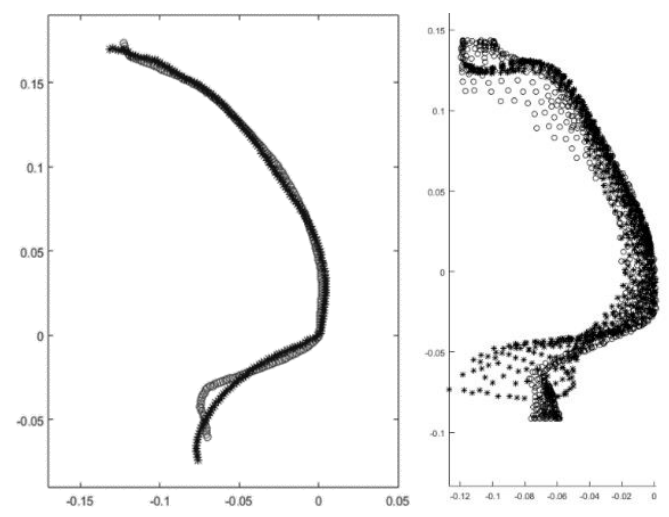

Fig. 6. Optimum fitting between the breast contour reconstructed from the MRI data (o) and the surface imaging of the breast $(*)$ after minimization of the objective function $f$ for the 2D model (left) and the 3D model (right) of the breast. We limit the nodes of the 3D finite elements model of the breast to the region within $+/-4 \mathrm{~cm}$ of the sagittal plane going through the nipple to help the visualization and comparison with the 2D model of the breast. Axis in meters. 
We then evaluated the objective function on the 3D breast contour, with a Young's modulus $E$ ranging from 0.5 to $2 \mathrm{kPa}$ with a resolution of $0.25 \mathrm{kPa}$; we then construct $k=10$ different 3D breast model from the Kinect data corresponding to different values of $b_{B}$. An illustration of a subset of those models are shown in Fig. 7. We obtain a global minimum of the objective function $f$ for $E=0.75 \mathrm{kPa}$, comparable to the result obtained with the 2D formulation of the problem.

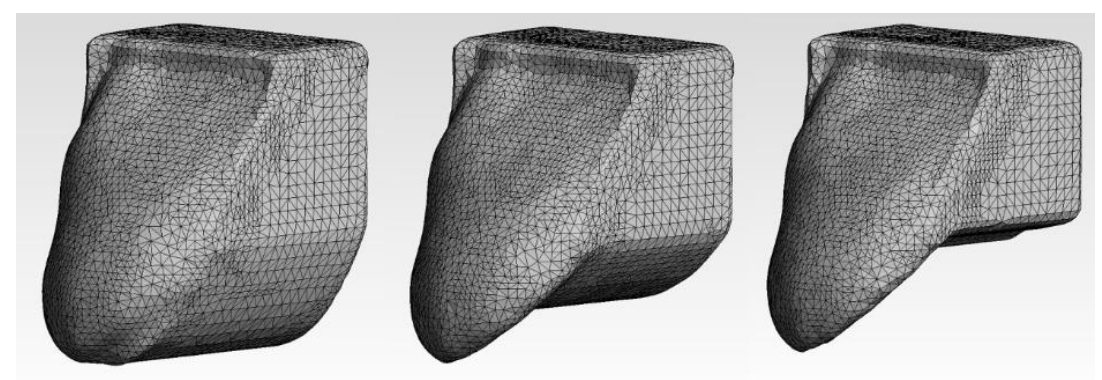

Fig. 7. Subset of the $k=103 \mathrm{D}$ breast model reconstruction from the Kinect surface imaging of the breast obtained by varying the constraining the parametric point $b_{B}^{k}$, corresponding to different lower surface of the breast.

\section{Conclusion}

We have detailed here a method making use of non-invasive, multimodal imaging of the breast of BCT patients that can successfully be used to accurately estimate simultaneously retrieve the breast elastic modulus, a critical parameter of our multiscale model, as well as the missing data of the breast contour when acquired with either MRI or surface imaging. This technique was easily extended to a three-dimensional analysis of the MRI and surface imaging data acquired in our clinical study with good results. We also plan to compare our estimation of the elastic modulus of the breast tissues with elastography measurements in order to complete the validation of our model.

The methods presented here provide a simple and non-invasive way to reconstruct a $3 \mathrm{D}$, patient-specific reference model of the breast and will help further validate our model of the outcome of breast conserving therapy, in order to provide clinically relevant insights to both patients and surgeons.

Acknowledgements. The authors would like to thank the Department of Surgery at the Houston Methodist hospital and the patients enrolled in the clinical trial, the Horizon 2020 grant Desiree (Award\# 690238) and all of its partners. 


\section{References}

1. Jemal, A., Siegel, R., Ward, E., Hao, Y., Xu, J., Murray, T. and Thun, M.J., 2008. Cancer statistics, 2008. CA: a cancer journal for clinicians, 58(2), pp.71-96.

2. Ma, J. and Jemal, A., 2013. Breast cancer statistics. In Breast Cancer Metastasis and Drug Resistance (pp. 1-18). Springer New York.

3. Van Dongen, J.A., Bartelink, H., Fentiman, I.S., Lerut, T., Mignolet, F., Olthuis, G., Van der Schueren, E., Sylvester, R., Winter, J. and Van Zijl, K., 1991. Randomized clinical trial to assess the value of breast-conserving therapy in stage I and II breast cancer, EORTC 10801 trial. Journal of the National Cancer Institute. Monographs, (11), pp.15-18.

4. Engel, J., Kerr, J., Schlesinger-Raab, A., Sauer, H. and Hölzel, D., 2004. Quality of life following breast-conserving therapy or mastectomy: Results of a 5-year prospective study. The breast journal, 10(3), pp.223-231.

5. van Dongen, J.A., Voogd, A.C., Fentiman, I.S., Legrand, C., Sylvester, R.J., Tong, D., van der Schueren, E., Helle, P.A., van Zijl, K. and Bartelink, H., 2000. Long-term results of a randomized trial comparing breast-conserving therapy with mastectomy: European Organization for Research and Treatment of Cancer 10801 trial. Journal of the National Cancer Institute, 92(14), pp.1143-1150.

6. Morris, A.D., Morris, R.D., Wilson, J.F., White, J., Steinberg, S., Okunieff, P., Arriagada, R., Lê, M.G., Blichert-Toft, M. and Van Dongen, J.A., 1996. Breast-conserving therapy vs mastectomy in early-stage breast cancer: a meta-analysis of 10-year survival.

7. Clough, K.B., Cuminet, J., Fitoussi, A., Nos, C. and Mosseri, V., 1998. Cosmetic sequelae after conservative treatment for breast cancer: classification and results of surgical correction. Annals of plastic surgery, 41(5), pp.471-481.

8. Veiga, D.F., Veiga-Filho, J., Ribeiro, L.M., Archangelo-Junior, I., Balbino, P.F., Caetano, L.V., Novo, N.F. and Ferreira, L.M., 2010. Quality-of-Life and Self-Esteem Outcomes after Oncoplastic Breast-Conserving Surgery [Outcomes Article]. Plastic and reconstructive surgery, 125(3), pp.811-817.

9. Garbey, M., Salmon, R., Thanoon, D. and Bass, B.L., 2013. Multiscale modeling and distributed computing to predict cosmesis outcome after a lumpectomy. Journal of Computational Physics, 244, pp.321-335.

10. Salmon, R., Garbey, M., Moore, L.W. and Bass, B.L., 2015. Interrogating a multifactorial model of breast conserving therapy with clinical data. Plos one, 10(4), p.e0125006.

11. Yeh, E.D., Georgian-Smith, D., Raza, S., Bussolari, L., Pawlisz-Hoff, J. and Birdwell, R.L., 2014. Positioning in breast MR imaging to optimize image quality. Radiographics, 34(1), pp.E1-E17.

12. Harvey, J.A., Hendrick, R.E., Coll, J.M., Nicholson, B.T., Burkholder, B.T. and Cohen, M.A., 2007. Breast MR Imaging Artifacts: How to Recognize and Fix Them 1. Radiographics, 27(suppl_1), pp.S131-S145.

13. Lepoutre, N., Gilles, M., Salmon, R., Collet, C., Bass, B., and Garbey, M., 2014. A robust method and affordable system for the 3D-surface reconstruction of patient torso to evaluate cosmetic outcome after Breast Conservative Therapy. Journal of Computational Surgery, $1(1), 11$.

14. Rajagopal, V., Chung, J.H., Bullivant, D., Nielsen, P.M. and Nash, M.P., 2007. Determining the finite elasticity reference state from a loaded configuration. International Journal for Numerical Methods in Engineering, 72(12), pp.1434-1451.

15. Rajagopal, V., Nash, M.P., Highnam, R.P. and Nielsen, P.M., 2008, July. The breast biomechanics reference state for multi-modal image analysis. In International Workshop on Digital Mammography (pp. 385-392). Springer Berlin Heidelberg. 
16. Recfusion, http://recfusion.net/index.php/en/

17. Multi-scale Modeling of Breast Conserving Therapy (BCT), https://clinicaltrials.gov/ct2/show/NCT02310711

18. Maas, S.A., Ellis, B.J., Ateshian, G.A. and Weiss, J.A., 2012. FEBio: finite elements for biomechanics. Journal of biomechanical engineering, 134(1), p.011005.

19. Thanoon, D., Garbey, M., Kim, N.H. and Bass, B., 2010. A computational framework for breast surgery: application to breast conserving therapy. In Computational Surgery and Dual Training (pp. 249-266). Springer US.

20. Cignoni, P., Callieri, M., Corsini, M., Dellepiane, M., Ganovelli, F. and Ranzuglia, G., 2008. Meshlab: an open-source mesh processing tool. Eurographics Italian Chapter Conference, 2008, pp. 129-136.

21. Geuzaine, C. and Remacle, J.F., 2009. Gmsh: A 3-D finite element mesh generator with built-in pre-and post-processing facilities. International Journal for Numerical Methods in Engineering, 79(11), pp.1309-1331.

22. Rzymski, P., Skórzewska, A., Skibińska-Zielińska, M. and Opala, T., 2011. Factors influencing breast elasticity measured by the ultrasound Shear Wave elastography-preliminary results. Archives of Medical Science, 7(1), pp.127-133. 